\title{
MANIPULATION OF BULK REACTIONS IN MULTIPHASE SYSTEMS BY THE USE OF MICROHETEROGENEOUS MEDIA
}

\author{
S. PATEL and A. MEHRA \\ Department of Chemical Engineering, Indian Institute of Technology, Bombay, Powai, Bombay, India
}

\begin{abstract}
I $\mathrm{t}$ is well known that the rates of fast, multiphase reactions, that occur in the diffusion zone near the interface, may be enhanced by the use of an additional, emulsified phase, which shows a high affinity for the rate limiting solute. However, the effect of such an additional phase on the rate behaviour of systems where slow reaction occurs dominantly in the bulk, well mixed region has not been assessed so far. This effect can be of great significance, especially in situations, where the reaction kinetics depend upon the reactant concentration in a complex way (e.g. substrate inhibition) and may be of particular importance in biochemical systems. In this study, the conversion behaviour of a model steroid system, namely, the biphasic, enzymatic conversion of testosterone in a batch reactor, has been explored in the presence of an emulsified oil phase. The kinetic rate of reaction of this species displays a maximum with respect to its concentration. It has been shown that the presence of an emulsified liquid phase can improve the substrate conversions significantly and that the extent of such an improvement depends upon the solubilizing capacity of this phase as well as its hold-up. The use of a microheterogeneous media, in lieu of a plain reactive liquid, can therefore be used to drive the reactor towards optimal behaviour with a view to maximizing the substrate conversions.
\end{abstract}

Keywords: multiphase; reaction; diffusion; emulsions

\section{INTRODUCTION}

The reaction between species that reside predominantly in different phases is usually brought about by contacting these phases in a multiphase reactor. For the reaction to occur, at least one of the reactants must be transported from its original phase to the other one where the reaction takes place. The interphase rates of mass transfer of this solute are limited by its sparing solubility in the reaction medium and are influenced by the diffusion coefficient of the reactant, the agitation intensity and the reaction rate constant. Doraiswamy and Sharma ${ }^{1}$ have provided a comprehensive theory of mass transfer accompanied by chemical reaction wherein such systems have been classified into various regimes. Broadly speaking, 'slow' reactions occur in the bulk of the receiving phase, subsequent to the transfer of the reactant across an interfacial ('film') layer which contains all the diffusional gradients. 'Fast' reactions, on the other hand, take place entirely in this diffusion zone and very little or none of the diffusing reactant ever reaches the bulk liquid. In between these two categories lie reactions that will occur in the film as well as the bulk.

In this context, the presence of an additional liquid phase in the form of emulsified droplets, which is immiscible with the original liquid phase and which shows a much higher affinity for the diffusant as compared to the original liquid, is known to enhance the rate of interphase mass transfer. This has been shown to be true for gas-liquid as well as solid-liquid systems ( Mehra et al. ${ }^{2}$, Bruining et al. ${ }^{3}$ and Van Ede et al. ${ }^{4}$ ). This strategy has been experimentally validated over a wide variety of systems and various theoretical approaches are available which encompass a large range of scenarios $\left(\mathrm{Mehra}^{5}\right.$; Nagy and $\mathrm{Moser}^{6}$ ).

However, the focus in most of the prior literature in this context has been on enhancing the interphase mass transfer flux across the interface formed by the original phases, and not on the behaviour of the bulk phase concentration of the diffusant. Most of the systems that have been studied involve fast reaction so that either a significant amount of the reaction occurs in the diffusion zone itself or if the locale of the reaction lies in the bulk then the reactant concentrations here tend to zero (in the absence and presence of the emulsified phase). In fact, some investigators deliberately chose operating conditions to ensure a near-zero bulk concentration so as to focus exclusively on the flux behaviour $^{3,4}$. The impact of the microdispersed phase on bulk phase reactions has never been assessed and this is a critical gap in the literature in this area. Moreover, all the previous studies deal with pseudo first order or second order kinetics.

In this study the effect of an additional, emulsified liquid phase, which shows a much higher affinity for the substrate as compared to the original liquid, on the behaviour of slow reactions that occur predominantly in the bulk phase and where the reactant concentration is finite is explored. This aspect can be of tremendous importance if the reaction kinetics depends upon the reactant concentration in a 
complex way (e.g. substrate inhibition). An analysis of this kind may be expected to provide valuable inputs for process design and development especially for biochemical systems where complex kinetics are frequently encountered.

\section{MODEL SYSTEM}

The enzymatic transformation of substrates, which are sparingly soluble in the enzyme bearing (aqueous) phase, may be typically carried out in multiphase reactors. These systems are usually rate limited by the diffusion of the substrate from the phase in which it is stored into the reactive (aqueous) medium. Instances of such transformations include the enzymatic conversion of steroid and hormone moieties [e.g. testosterone to androst-4-ene-3,17dione (Bhasin et al..$^{7}$ ] as well as the hydrolysis of high molecular weight fats [e.g. lipase catalysed, olive oil to oleic acid (Tsai et $\left.a l_{.}^{8}\right)$ ]. In some cases the reaction product(s) too may be sparingly soluble in the aqueous phase.

The rationale for using a multiphase contacting strategy, as opposed to a single aqueous phase system, is based on the arguments that (i) very large aqueous volumes (and correspondingly, large amounts of the dissolved enzyme) are required in a single phase, homogeneous system, on account of the dilute concentrations of the substrate and (ii) there usually occur downstream separation/recovery problems associated with the removal of these low concentration solutes.

A variety of multiphase solutions have been suggested in the literature for obviating these problems. Cremonsei et al. ${ }^{9}$ and later, Boeren and Laane ${ }^{10}$ proposed the idea of dissolving the hydrophobic substrate in a water-immiscible solvent (oil) followed by the dispersion of this substrate reservoir in the aqueous, enzymatic containing phase. The former investigators also studied the effect of various organic solvents on enzyme activity and concluded that the enzymatic activity decreased with increasing polarity of the solvent while the solubility of the substrate showed the reverse trend. It was shown that butyl acetate was the best solvent from an overall, conversion point of view. Similarly, Boeren and Laane ${ }^{10}$ demonstrated for a slightly different but related system that the use of an octane-water combination produced better yields compared to a purely aqueous reactor. Thus even though enzymes may be subject to some denaturation in the presence of an organic solvent, the overall impact may still be favourable. Another alternative, namely, the use of polymeric resins as reservoirs for hydrophobic substrates was first suggested by Bhasin et al. ${ }^{7}$ who reported the occurrence of enzyme denaturation due to the presence of the organic solvent. The use of polymeric solids as substrate reservoirs, in lieu of organic solvent drops, was therefore proposed in order to avoid the danger of enzyme denaturation and also to provide for easier handling compared to oil-in-water emulsions. Pereira et al. ${ }^{11}$ actually studied the strategy of loading the sparingly water-soluble substrate on to a hydrophobic polymer and the subsequent suspension of these loaded polymer beads in the aqueous enzyme solution. These workers examined the conversion of testosterone $(17 \beta-$ hydroxyandrost-4-ene-3-one) to androst-4-ene-3,17-dione by the enzyme 3,17 $\beta$-hydroxysteroid dehydrogenase (recovered from Pseudomonas testosteroni).

In this study the testosterone conversion system mentioned above is considered. The substrate was loaded on beads of polydimethylsiloxane (PDMS) which were suspended in an aqueous enzyme solution (also containing a co-factor) and the whole suspension operated in batch mode. The testosterone, which has low solubility in the aqueous phase, diffuses from the polymeric beads into the aqueous phase where it undergoes enzymatic transformation to the product which itself diffuses back into the polymer material. The aqueous phase (intrinsic) reaction kinetics for this system is strongly substrate inhibited so that there occurs an optimum concentration at which the rate of reaction is maximum. Also, the size and concentration of the polymer beads may be used to regulate substrate concentration levels in the enzymatic phase in order to maximize the yield of the product. The diffusion-reaction process here is inherently unsteady since the concentration profiles of the substrate in the PDMS (reservoir) beads, the interfacial substrate concentration at the PDMS-liquid interface and the bulk substrate concentration, all vary with batch time.

For a system with such substrate inhibited kinetics, it is desirable to control the substrate concentration to near optimal levels so that low transformation rates that will prevail at low and high substrate concentrations can be avoided. It may be possible to achieve this objective by emulsifying an oil phase in the enzymatic (aqueous) phase, which is capable of solubilizing a substantial amount of the substrate. The extent of such 'doping' by a dispersed phase, and its solubilizing capacity, will therefore affect the substrate levels in the reactive (aqueous) phase. The effect of an oil phase, in such a system, on the substrate conversion, is quantitatively explored in this work.

It needs to be pointed out that any benefits obtained in the substrate conversions through the use of the strategy proposed in this study would ultimately have to be weighed against the additional costs of downstream processing requirements-breaking the emulsion, product recovery from the organic phase (if the product is also lipophilic), etc.

\section{MODEL DESCRIPTION}

A schematic view of the diffusion-reaction process is shown in Figure 1. The system thus considered here

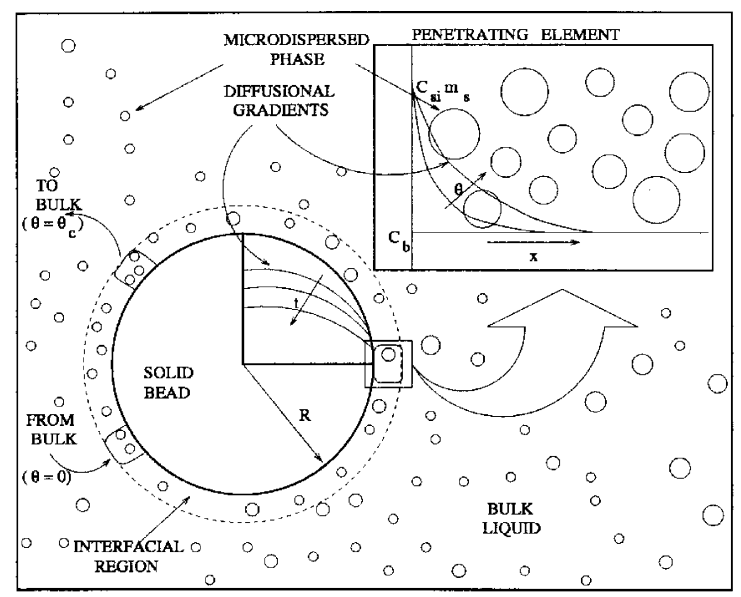

Figure 1. Schematic drawing of mass transport of the substrate from reservoir particle to bulk of the reactive liquid phase based on Higbie's mass transfer theory. 
comprises of solid beads of radius, $R$ in which the substrate $A$ is initially present at a concentration, $C_{s o}$ and diffuses radially outwards to the surface, where it is taken up by the liquid phase containing the emulsified microdroplets, and in which these solid beads are suspended.

The essential action of the fine, emulsified droplets lies in the uptake of the diffusing substrate by the droplets in the interfacial, diffusion zone of the original liquid and the subsequent release of this extra solute into the bulk liquid phase, when an interfacial liquid element returns to the bulk from the interface. A necessary condition for this mechanism to be operative is that the size of the emulsified droplets should be less than the diffusion length scales of the substrate in the original liquid phase $\left(d_{p} \ll D_{l} / k_{l}\right)$. This 'consumption' of the substrate in the interfacial region results in an increase in the steepness of the concentration gradients and a consequent rise in the interphase transfer flux of the substrate. The release of the substrate, loaded within these drops, into the bulk may be expected to increase the bulk concentration of the substrate while the extra capacity to store this substrate in the droplets present in the bulk should create a reverse tendency. Overall, the bulk concentration of the substrate is likely to be lower, compared to the case when the emulsion is absent, during the initial period in a batch reactor when the concentration is rising from its initial value (usually, zero) and should be higher in the later period when the concentration is decaying due to chemical reaction. In the former scenario, the droplets act as storage sinks whereas in the latter case these act as sources.

Since there is no reaction taking place within the solid phase, the equation for transport of the substrate within a single reservoir sphere may be written as

$$
\frac{\partial C_{s}}{\partial t}=\frac{D_{s}}{r^{2}} \frac{\partial}{\partial r}\left(r^{2} \frac{\partial C_{s}}{\partial r}\right)
$$

with boundary conditions

$$
\begin{aligned}
& \left(\frac{\partial C_{s}}{\partial r}\right)_{r=0}=0 \\
& -D_{s}\left(\frac{\partial C_{s}}{\partial r}\right)_{r=R}=q_{i}(t)
\end{aligned}
$$

where, $q_{i}(t)$ is the flux of the substrate at the solid-liquid interface, and the initial condition

$$
C_{s}(r, 0)=C_{s o}
$$

For the purpose of describing solid-liquid mass transfer Higbie's mass transfer model was chosen. In this picture, the interfacial liquid layer is deemed to comprise of a mosaic of penetration elements which spend a fixed time $\theta_{c}$ at the interface. At the end of this time period these elements return to the bulk liquid and are replaced at the interface by fresh ones arriving from the bulk. This continuing process of penetration elements shuttling between the interface and the bulk region is thus a representation of convective mixing in the reactor ${ }^{1,12}$. The relationship between the contact time, $\theta_{c}$ and the measured physical mass transfer coefficient, $k_{l}$ is given by

$$
\theta_{c}=\frac{4 D_{l}}{\pi k_{l}^{2}}
$$

The mass transfer coefficient, $k_{l}$ depends upon the stirring intensity, bead size, the diffusion coefficient, $D_{l}$ and other fluid properties. In order to properly demonstrate the effect of stirring intensity or the bead size (which in turn also causes the $k_{l}$ value to change) on the reactor behaviour, it is necessary to use a typical empirical correlation for stirred reactors which relates $k_{l}$ to $R$ and other relevant physicochemical parameters. The correlation given by Kubai et al. ${ }^{13}$ was chosen for this purpose, namely

$$
S h=2+0.49\left(R e_{p} S c\right)^{\frac{1}{2}}
$$

where $S h=k_{l}(2 R) / D_{l}, S c=v / D_{l}$ and $R e_{p}$, based on the slip velocity between the solid and liquid, is defined as

$$
R e_{p} \sim \frac{1}{2}\left(\frac{\varepsilon(2 R)^{4}}{\rho v^{3}}\right)^{\frac{1}{3}}
$$

where, $v=\mu / \rho$ and $\varepsilon$ is the energy dissipation per unit volume of the liquid phase. The advantage of using this correlation is that it is independent of the kind of impeller used and depends solely upon the energy dissipation per unit volume of the liquid phase.

The process of simultaneous diffusion-reaction in a penetration element in the presence of an emulsified 'sink' phase is defined $b^{5}$

$$
F \frac{\partial C_{l}}{\partial \theta}=D_{l} \frac{\partial^{2} C_{l}}{\partial x^{2}}-(1-l) r_{c}\left(C_{l}\right)
$$

where, $r_{c}$ is the reaction rate, and is given by

$$
r_{c}\left(C_{l}\right)=V_{m} /\left(1+K\left(\frac{C_{o}}{C_{l}}+\frac{C_{l}}{C_{o}}\right)\right)
$$

for the strongly substrate inhibited system considered here (Marcus and Talalay ${ }^{14}$, referred to hereafter as MT kinetics). This kinetic expression indicates that the rate of liquid phase reaction is maximum at the optimum concentration, $C_{o}$. The initial condition is given by

$$
C_{l}(x, 0)=C_{b}
$$

and the boundary conditions are

$$
\begin{aligned}
& C_{l}(0, \theta)=C_{s i}(t) / m_{s} \\
& C_{l}(\infty, \theta)=f(\theta)
\end{aligned}
$$

The first boundary condition [equation (11)] provides for concentration continuity at the solid-liquid interface. Here, $C_{s i}$ is the solid phase concentration of the substrate at the solid-liquid interface and $m_{s}$ denotes the partition coefficient of the substrate between the solid and the liquid phases. $F=(1-l)+l m_{d}$ and is a measure of the combined capacity of the original liquid phase as well as the microdispersed phase to store the substrate; $l$ is the fractional, volumetric hold-up of the emulsified phase and $m_{d}$ is the partition coefficient for the substrate between the original liquid and the microdispersed liquid. Also, $f(\theta)$ is the solution of the above material balance [equation (8)] without the diffusive term. This condition essentially states that 'far away' from the interface the reactant concentration decays due to reaction and uptake by the emulsified phase droplets according to the function $f(\theta)$ starting from the initial value of $C_{b}$ (Danckwerts ${ }^{12}$ ). The initial condition [equation (10)] implies that an element that has just arrived at the interface from the bulk carries within it conditions that prevail in the bulk liquid. For this formulation to be vallid, $\theta_{c}$ must be much smaller than the 
time scale of diffusion in the solid bead, i.e. $R^{2} / D_{s}$ as well as the batch time scale. These conditions are valid for all the computations carried out in this study and are required to be satisfied so that the boundary condition stated by equations (11) and (12) change insignificantly over the period $\theta_{c}$.

The flux of the substrate from the solid phase into the liquid elements, averaged over the entire solid-liquid interface, is now given by the time-average over $\theta_{c}$, namely

$$
q_{i}=\frac{1}{\theta_{c}} \int_{0}^{0_{c}}-D_{l}\left(\frac{\partial C_{l}}{\partial x}\right)_{x=0} \mathrm{~d} \theta
$$

while the flux of substrate transferred to the bulk phase from the interfacial region, due to shuttling of the penetration elements is defined by ${ }^{2}$

$$
q_{b}=\frac{F}{\theta_{c}}\left[\int_{0}^{\infty}\left(C_{l}\left(\theta_{c}, x\right)-C_{l}(0, x)\right) \mathrm{d} x\right]
$$

where the integral on the right hand side of equation (14) represents the amount transferred to the bulk by elements moving out from the interfacial zone (carrying within them the conditions that prevail at time $\theta_{c}$ ) minus the material brought into the interfacial zone by a fresh element arriving from the bulk. Thus the rate at which substrate enters the liquid phase $\left(q_{i}\right)$ is different from the rate at which it enters the bulk liquid $\left(q_{b}\right)$; the difference being on account of the amount that has reacted in the interfacial region near the solid boundary.

The bulk material balance for the substrate concentration is written as

$$
F \frac{\mathrm{d} C_{b}}{\mathrm{~d} t}=a_{p} q_{b}(t)-(1-l) r_{c}\left(C_{b}\right)
$$

where, $a_{p}$ is the specific solid-liquid interfacial area given by

$$
a_{p}=\frac{3 V_{s}}{R V_{l}}
$$

The relevant initial condition for equation (15) is

$$
C_{b}(0)=0
$$

The output quantities of interest, namely, the average concentration of the substrate in a polymer bead, $\left\langle C_{s}\right\rangle$ and the fractional substrate conversion, $X$ are given by

$$
\left\langle C_{s}\right\rangle(t)=\frac{3}{R^{3}} \int_{0}^{R} r^{2} C_{s}(r, t) \mathrm{d} r
$$

and

$$
X(t)=1-\frac{\left\langle C_{s}\right\rangle}{C_{s o}}-\frac{V_{l} F C_{b}}{V_{s} C_{s o}}
$$

respectively.

The formulation is now complete and the above set of equations can be solved to obtain $C_{s i}, q_{i}, q_{b}$ and $C_{b}$ (and hence also, $\left\langle C_{s}\right\rangle$ and $X$ ) versus batch time, $t$. The dimensionless versions of these equations were solved numerically. Equation (1), for diffusion within the solid phase, was discretized in the radial coordinate using orthogonal collocation (Villadsen and Michelsen ${ }^{15}$ ) to yield a system of ordinary differential equations (ODEs) in time. The flux condition at $r=R$ results in a non-linear algebraic equation which has to be solved iteratively to obtain the unknown interface concentration, $C_{s i}$. This mixed system of these ODEs and algebraic equations, along with equation (15), which describes the evolution of the bulk concentration, were integrated in time $(t)$ using the Livermore solver for ordinary differential equations (LSODE; Radhakrishnan and Hindmarsh ${ }^{16}$ ). To evaluate $q_{i}$ and $q_{b}$, the equations describing the diffusion-reaction within the penetrating element [equations (8), (10), (11) and (12)] were solved by discretizing the spatial terms using finite differences. The resulting system of ODEs was integrated in time $(\theta)$ using the Crank-Nicholson algorithm. A fixed point iterative scheme was employed to deal with the non-linearities associated with $r_{c}$ because the kinetic expression used was non-linear, i.e. the Marcus-Talalay kinetics given by equation (9). For computational purposes, the spatial domain on $x$, extending from 0 to $\infty$, was taken to be of increasing, finite lengths such that the solutions were no longer dependent on this length. The integrals in equations (13) and (14) were evaluated using the trapezoidal rule. The integral in equation (18) was evaluated using the Gauss-Jacobi quadrature.

For the case when all reaction occurs exclusively in the bulk, it can be shown that ${ }^{5}$

$$
q_{i}=q_{b}=\sqrt{F} k_{l}\left(C_{s i} / m_{s}-C_{b}\right)
$$

This amounts to solving equation (8) analytically after neglecting the reaction term. The conditions for the reaction to occur predominantly in the bulk phase are given by ${ }^{1}$

$$
\begin{aligned}
H a & =\sqrt{k_{e} D_{l}} / k_{l} \ll 1 \\
D a & =k_{e} /\left(k_{l} a_{p}\right) \gg 1
\end{aligned}
$$

where, $H a$ and $D a$ are the Hatta and Damkohler numbers, respectively, and $k_{e}$ is an effective first order rate constant. For the MT kinetics the maximum value of $k_{e}$ may be taken to be

$$
k_{e}=\max \left[\frac{r_{c}}{C_{l}}\right]=V_{m} / K C_{o}
$$

The values of all parameters used in this study are listed in Table 1. For these values, the maximum $H a=0.6$ and the maximum $D a=195$. Clearly, our system can also be described by the simplifications mentioned above.

Table 1. Parameters used in this study.

\begin{tabular}{lll}
\hline Parameter & \multicolumn{1}{c}{ Value } & \multicolumn{1}{c}{ Source } \\
\hline$C_{o}$ & $6.0 \mathrm{mmol} \mathrm{m}^{-3}$ & Pereira et al. $(1987)^{11}$ \\
$C_{s o}$ & $4.0 \times 10^{3} \mathrm{mmol} \mathrm{m}^{-3}$ & $*$ Pereira et al. $(1987)^{11}$ \\
$D_{l}$ & $5.6 \times 10^{-10} \mathrm{~m}^{2} \mathrm{~s}^{-1}$ & Wilke and Chang $(1955)^{17}$ \\
$D_{s}$ & $6.0 \times 10^{-11} \mathrm{~m}^{2} \mathrm{~s}^{-1}$ & Michaels et al. $(1975)^{18}$ \\
$K$ & 0.154 & Pereira et al. $(1987)^{11}$ \\
$m_{s}$ & 20 & $*$ Pereira et al. $(1987)^{11}$ \\
$m_{d}$ & 20 & $*$ Bhasin et al. $(1987)^{11}$ \\
$V_{m}$ & $3.0 \times 10^{-2} \mathrm{mmol} \mathrm{m}^{-3} \mathrm{~s}^{-1}$ & $*$ Pereira et al. $(1987)^{11}$ \\
$\rho$ & $1.0 \times 10^{3} \mathrm{~kg} \mathrm{~m}^{-3}$ & $* *$ \\
$\mu$ & $1.0 \times 10^{-3} \mathrm{~Pa} \mathrm{~s}^{-3}$ & $* *$ \\
$\varepsilon$ & $1.0 \times 10^{-2} \mathrm{~W} \mathrm{~m}^{-3}$ & $* * *$ \\
\hline
\end{tabular}

* Values are of the same order of magnitude as suggested here.

$* *$ For water.

*** Typically estimated value for a mildly stirred reactor. 
However, this simplified model needs to be used with caution because a slight change in the operating parameters can push the system, at least partially, into the interfacial (fast) reaction regime where the rigorous version needs to be used $\left(q_{i} \neq q_{b}\right)$.

\section{RESULTS AND DISCUSSION}

\section{Basic System Behaviour: the Importance of Bulk Substrate Concentration}

The overall conversion, $X$ versus particle radius, $R$, for different values of the liquid to solid volume ratio, $V_{l} / V_{s}$ and maximum reaction velocity, $V_{m}$, is shown in Figure 2, in the absence of the additional, emulsified phase. At small $R$, the bulk concentration, $C_{b}$ builds up very fast to values which are higher than $C_{o}$, leading to low reaction rates. The bulk concentration thus remains high for a large time as seen in the inset for particles of $R=2 \mathrm{~mm}$. An increase in $R$ leads to a slower build up of $C_{b}$; this now remains near $C_{o}$ for quite some time (see inset for $R=3 \mathrm{~mm}$ ) causing a higher conversion of the substrate. As the particle radius is increased further, a point is reached when the conversion attains a maximum, after which any increase in $R$ now forces $C_{b}$ to values much lower than $C_{o}$ (see inset for $R=4 \mathrm{~mm}$ ). In this region the reaction rates are directly proportional to the substrate concentrations. Thus, there exists an optimal radius, for MT kinetics, as was first suggested by Pereira et al. ${ }^{11}$. It may also be noticed that larger particles offer greater internal resistance to diffusion so that the substrate concentration at the solid-liquid interface, $C_{s i} / m_{s}$ declines more rapidly with time for a large particle as compared to a small one. The intersection

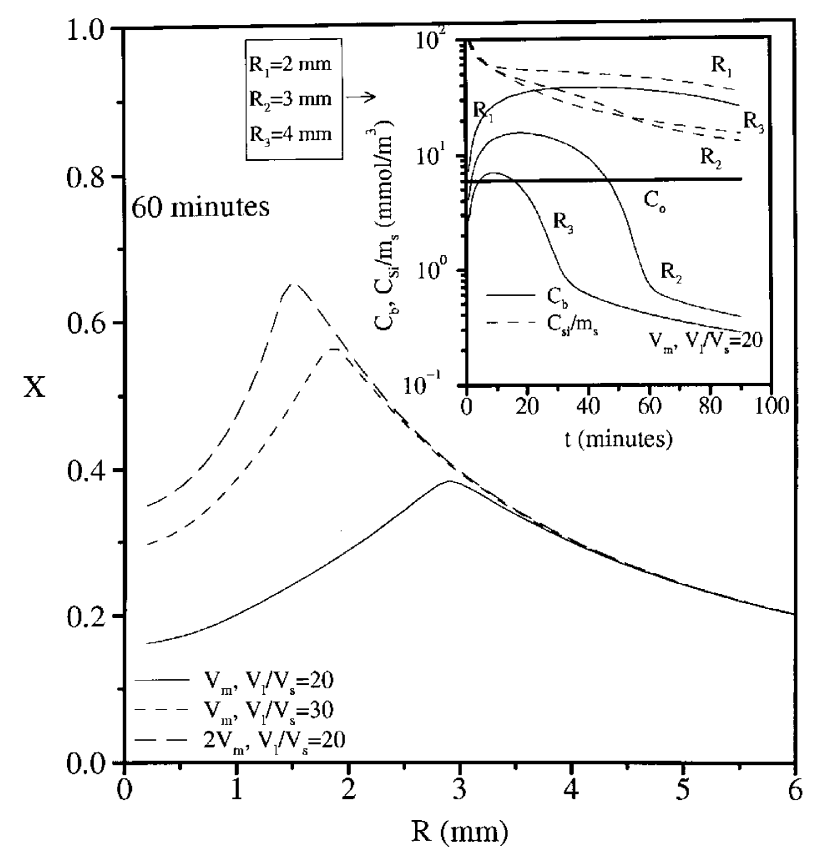

Figure 2. Substrate conversion, $X$ versus bead size, $R$ for Marcus-Talalay (MT) kinetics, in a batch reactor at $t=60 \mathrm{~min}$, in the absence of an emulsified phase. Parameters varied - reaction velocity, $V_{m}$ and liquid to solid volume ratio, $V_{l} / V_{s}$. Inset shows variation of the bulk substrate concentration, $C_{b}$ and the substrate concentration at the solid-liquid interface, $C_{s i} / m_{s}$ with time, $t$ at three values of $R$ and $V_{l} / V_{s}=20$. Data used is listed in Table 1. between the plots for $C_{s i} / m_{s}$ versus $t$, in the inset, for $R_{2}$ and $R_{3}$, is on account of the reaction rates becoming higher for the former size at later times since $C_{b}$ for this size is closer to $C_{o}$.

An increase in the liquid to solid volume ratio, $V_{l} / V_{s}$ will decrease the interfacial area (per unit volume of liquid) and hence lower the overall mass transfer rates from the solid to the bulk liquid but will increase the total volume for reaction. Thus, even though the $C_{b}$ values are lowered, in the range of small $R$, the larger liquid volume available for reaction more than compensates leading to higher conversions for a reaction that occurs predominantly in the bulk. Additionally, the conversions at small $R$, are also influenced by the increase in the reaction rate due to decrease in the bulk concentrations which are now brought closer to $C_{o}$. Therefore, at large $V_{l} / V_{s}$, smaller particles are required to maintain the concentration close to $C_{o}$; hence the maximum in $X$ shifts to the left. As $R$ is increased further, $C_{b}$ falls much below the $C_{o}$ and the reaction rates decline. Now, $C_{b}$ is lowered in direct proportion to the increase in $V_{l} / V_{s}$. The condition $C_{b} \ll C_{s i} / m_{s}$ is satisfied and so

$$
q_{i}=q_{b}=k_{l} C_{s i} / m_{s}
$$

becomes valid. This may be seen from the diminishing impact of a change in the liquid volume, $V_{l}$ on $X$ at larger $R$.

The effect of an increase in the value of $V_{m}$ is similar to that of an increase in the ratio, $V_{l} / V_{s}$ and this is also shown in Figure 2. The maximum in $X$ again shifts to the left indicating that equation (24) is now governing.

\section{System Behaviour in the Presence of an Emulsified Phase: Manipulation of Bulk Substrate Concentration}

The system behaviour for MT kinetics, in the absence and presence of an emulsified oil phase, is shown in Figure 3, at $30,60,90$ and 120 minutes. When $R$ is small, $C_{b}$ surges to high values in a short time due to the high mass transfer rates. The emulsion helps to depress $C_{b}$ values which move closer to $C_{o}$ for the entire time course of operation. This is seen from the variation of $C_{b}$ with $t$ in the inset, for $R=2 \mathrm{~mm}$. Now consider the case shown for $R=3 \mathrm{~mm}$. For these particles, closer to the optimum size, the emulsion ensures greater proximity between $C_{b}$ and $C_{o}$ for most of the time. At short times, the presence of the emulsion depresses $C_{b}$ while at large times it elevates $C_{b}$, relative to the corresponding transient profile of $C_{b}$ in the absence of emulsion. It is pertinent to note that a decay phase in $C_{b}$ will occur only in the case of a finite reservoir scenario, where the interfacial substrate concentration $C_{s i} / m_{s}$ decreases with batch time. For the case of a constant $C_{s i} / m_{s}$ there will exist only a rise or build-up period towards a steady state. Whereas, this 'finiteness' of the reservoir is neither difficult to model nor of much significance by itself it is important that the concentration $C_{b}$ is lower during the rise phase and higher in the decay phase, in the presence of the emulsified oil than in its absence. Thus, during the rise as well as decay of the concentration $C_{b}$ with respect to time, the effect of the emulsion is to push its value closer to the optimum value $C_{o}$, leading to higher substrate conversions. Therefore, at sufficiently long times, the conversions are 


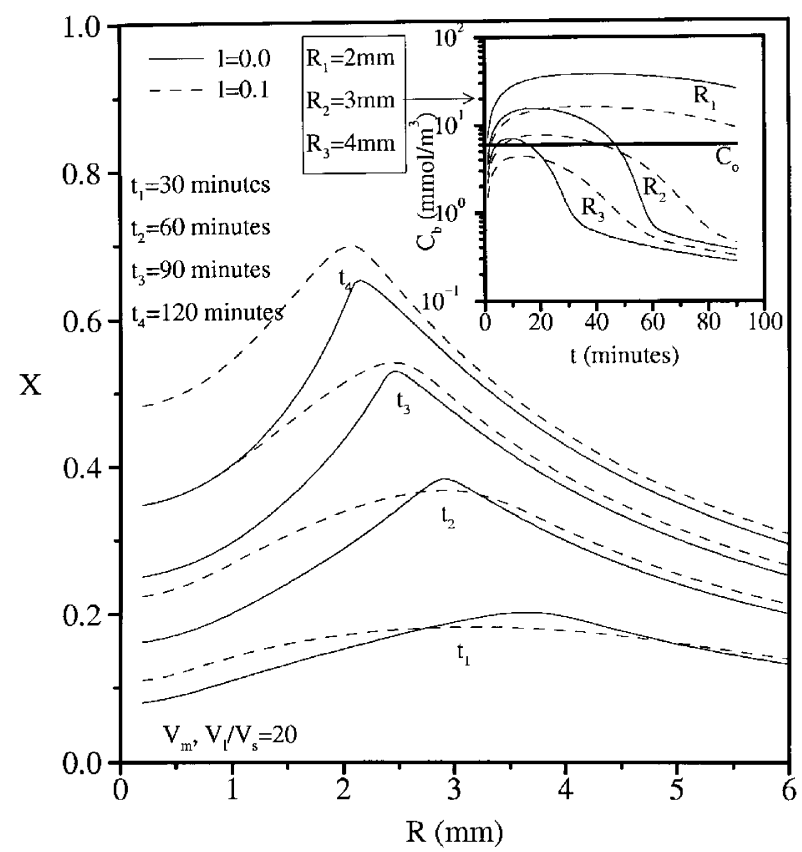

Figure 3. Substrate conversion, $X$ versus bead size, $R$ for Marcus-Talalay (MT) kinetics, in a batch reactor at $t=30,60,90$ and 120 minutes, in the presence of an emulsified phase and in its absence; $l=0.1, V_{l} / V_{s}=20$. Inset shows variation of the bulk substrate concentration, $C_{b}$ with time, $t$ at three values of $R$. Data used is listed in Table 1 .

higher at all particle sizes. There is, however, an initial time period when $C_{b}$ in the absence of emulsion is closer to $C_{o}$ than in its presence. For instance, the $C_{b}$ versus $t$ profile, in the inset of Figure 3, shows this to be true up to around 22 minutes at $R=4 \mathrm{~mm}$. This leads to $X$ being lower in the presence of the emulsion phase at 30 minutes, for $R=4 \mathrm{~mm}$; at 60 minutes $X$ has become higher when the emulsion is present and the impact of the initial period has been overcome. It can also be seen that at increasing times, the beneficial impact of the emulsion becomes more pronounced, especially for the smaller radii. Some typical increments in the conversion may be estimated from this figure; for $R=1 \mathrm{~mm}$ the substrate conversion increases from about $38 \%$ to about $55 \%$, due to the presence of the emulsion, at a batch time of 120 minutes, whereas this change is from $27 \%$ to $38 \%$ at 90 minutes.

Figure 4 shows the effect of the volume fraction of the emulsion on the substrate conversions. The lower frame in this figure gives the $X$ versus $R$ plots for different values of $l$. As the fractional hold-up of the emulsified phase is raised, the conversions first increase but then start decreasing. This happens because of the reduction in the relative volume of the reactive, aqueous phase. The top, right frame in this figure shows that every increase in $l$, over the full range up to $l=0.25$, pushes $C_{b}$ closer to $C_{o}$; indeed, as the top, left frame indicates, the reaction rates $\left(r_{c}\right.$ - thick lines) are highest when $l=0.25$, but when these are multiplied by the volumetric fraction of the aqueous phase, $(1-l)$ [which appears in equation (15)], the effective reaction rates are lowest for $l=0.25$. Thus at $R=2 \mathrm{~mm}, 10 \%$ of the emulsion phase is beneficial whereas $25 \%$ of it causes the advantage arising out of proximity between $C_{b}$ and $C_{o}$ to be over compensated by a reduction in reaction volume.
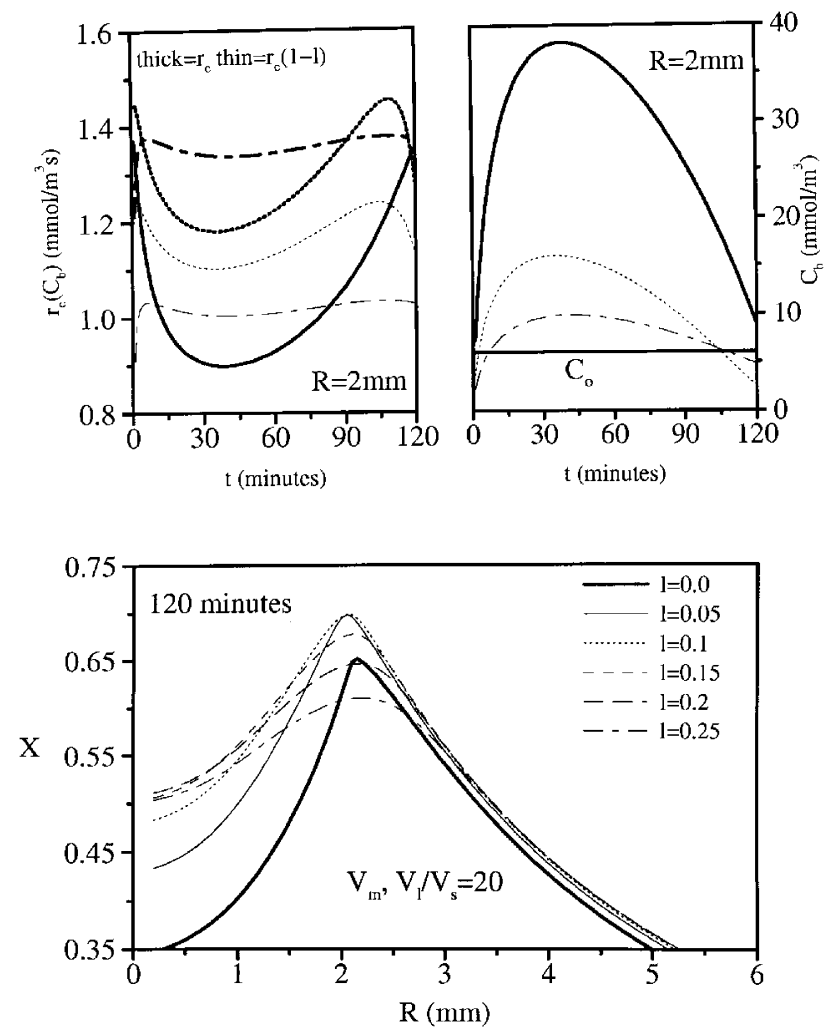

Figure 4. Substrate conversion, $X$ versus bead size, $R$ for Marcus-Talalay (MT) kinetics, in a batch reactor at $t=120$ minutes, at different fractional hold-ups of the emulsified phase (bottom frame); Top, right frame shows variation of the bulk substrate concentration, $C_{b}$ with time, $t$ at $R=2 \mathrm{~mm}$, while top, left frame shows bulk reaction rate, $r_{c}$ with time. Data used is listed in Table 1.

The basic feature of a maximum in the $X$ versus $R$ behaviour, for MT kinetics, remains even in the presence of the emulsion. Specifically, for small beads, when $C_{b}$ is high, the presence of emulsified droplets can be seen to lead to larger conversions by reducing the substrate overload in the reactive phase. Thus, an additional, emulsified liquid phase may be used as a means of controlling the system behaviour, especially towards maximizing the substrate conversion.

These results point to the possibility of a novel operating strategy, namely, the simultaneous use of oil and solid phases. Unlike, Bhasin et al. ${ }^{7}$ who exclusively used an oil phase as the substrate reservoir and Pereira et al. ${ }^{11}$ who used only polymer beads, a more optimal strategy may be to use both oil and solid reservoir phases in combination. For instance, it may be more feasible to add an emulsified phase to the system in order to attain greater conversions within a given time than to fabricate particles of a size different from those already available.

\section{CONCLUSIONS}

The effect of an emulsified, second (substrate-philic) liquid phase on the conversion behaviour of a multiphase batch reactor with substrate inhibited (Marcus-Talalay) biokinetics has been explored in this work. Here, the substrate is sparingly soluble in the aqueous (enzymatic) phase and is therefore stored in high affinity, polymeric reservoirs. Most of the reaction occurs within the bulk 
liquid phase and not in the diffusion zone surrounding the reservoir beads. The presence of the emulsion has been shown to be effective in increasing the substrate conversions, especially, for small particles.

Generally, the additional liquid phase can be used for modifying the transient behaviour of the bulk substrate concentration to achieve greater conversions. The emulsified droplets serve to depress the bulk concentration during the period of its rise and to elevate its value during the phase of reactive decay. This effect has not been explored previously for reactions that occur predominantly in the bulk phase. Prior studies have focused exclusively on fast reactions and the enhancement obtained in the rates of interphase mass transfer.

From the process design viewpoint, the practical significance of the multiphase contacting strategy proposed in this work is that it may be better to use a combination of solid and solvent reservoirs for the substrate rather than any of these phases alone. Such three-phase systems should be further explored in order to assess their viability as candidates for process development.

\section{NOMENCLATURE}

$a_{p} \quad$ interfacial area of solid per unit volume of liquid, $\mathrm{m}^{2} \mathrm{~m}^{-3}$

$C_{b} \quad$ bulk concentration in liquid phase, $\mathrm{mmol} \mathrm{m}^{-3}$

$C_{l}$ concentration in liquid phase, $\mathrm{mmolm}^{-3}$

$C_{o}$ optimum concentration in liquid phase, $\mathrm{mmol} \mathrm{m}^{-3}$

$C_{s} \quad$ concentration in solid (bead) phase, $\mathrm{mmol} \mathrm{m}^{-3}$

$C_{s i} \quad$ concentration at the solid-liquid interface in solid phase, $\mathrm{mmol} \mathrm{m}^{-3}$

$C_{s o}$ initial concentration in the solid phase, $\mathrm{mmol} \mathrm{m}^{-3}$

$d_{p} \quad$ emulsified phase droplet diameter, $\mathrm{m}$

Da Damkohler number, -

$D_{l} \quad$ diffusivity in liquid phase, $\mathrm{m}^{2} \mathrm{~s}^{-1}$

$D_{s} \quad$ diffusivity in solid phase, $\mathrm{m}^{2} \mathrm{~s}^{-1}$

$F \quad$ solubilizing capacity of continuous plus emulsified phase, -

$\mathrm{Ha}$ Hatta number, -

$K$ parameter in the MT rate expression, -

$k_{l} \quad$ liquid side mass transfer coefficient, $\mathrm{m} \mathrm{s}^{-1}$

$k_{e} \quad$ effective first order rate constant, $\mathrm{s}^{-1}$

$l \quad$ volume fraction of dispersed phase in liquid, $\mathrm{m}^{3} \mathrm{~m}^{-3}$

$m_{d}$ dispersed phase-liquid partition coefficient, -

$m_{s}$ solid-liquid partition coefficient, -

$q_{b} \quad$ flux into bulk liquid, $\mathrm{mmol} \mathrm{m}^{-2} \mathrm{~s}^{-1}$

$q_{i} \quad$ flux into liquid phase at solid-liquid interface, $\mathrm{mol} \mathrm{m}^{-2} \mathrm{~s}^{-1}$

$r$ radial coordinate in solid bead, $\mathrm{m}$

$r_{c} \quad$ reaction rate, $\mathrm{mmol} \mathrm{m} \mathrm{m}^{-3} \mathrm{~s}^{-1}$

$R$ particle (bead) radius, $m$

$\operatorname{Re}_{p}$ Reynolds number, -

Sc Schmidt number, -

Sh Sherwood number, -

$t$ time (batch scale), s

$V_{m}$ maximum reaction velocity (MT kinetics), $\mathrm{mmol} \mathrm{m}^{-3} \mathrm{~s}^{-1}$

$V_{l} \quad$ volume of liquid, $\mathrm{m}^{3}$

$V_{s} \quad$ volume of solid, $\mathrm{m}^{3}$

$x \quad$ space coordinate in penetration element, $\mathrm{m}$

$X$ substrate conversion, -

Greek letters

$\theta_{c} \quad$ Higbie's contact time, $\mathrm{s}$

$\rho \quad$ liquid density, $\mathrm{kg} \mathrm{m}^{-3}$

$\mu \quad$ liquid viscosity, Pas kinematic viscosity, $\mathrm{m}^{2} \mathrm{~s}^{-1}$

energy dissipation per unit volume, $\mathrm{W} \mathrm{m}^{-3}$

\section{REFERENCES}

1. Doraiswamy, L. K. and Sharma, M. M., 1984, Heterogeneous Reactions Vol 2, (John Wiley, New York).

2. Mehra, A., Pandit, A. and Sharma, M. M., 1988, Intensification of multiphase reactions through the use of microphase-II. Experimental, Chem Eng Sci, 43: 913-927.

3. Bruining, W. J., Joosten, G. E. H., Beenackers, A. A. C. M. and Hofman, H., 1986, Enhancement of gas-liquid mass transfer by a dispersed second liquid phase, Chem Eng Sci, 41: 1873-1877.

4. Van Ede, C. J., Van Houten, R. and Beenackers, A. A. C. M., 1995, Enhancement of gas to water mass transfer rates by a dispersed organic phase, Chem Eng Sci, 50: 2911-2922.

5. Mehra, A., 1988, Intensification of multiphase reactions through the use of microphase-1. Theoretical, Chem Eng Sci, 43: 899-912.

6. Nagy, E. and Moser, A., 1995, Three-phase mass transfer: improved pseudo-homogeneous model, AIChEJ, 41: 23-34.

7. Bhasin, D. P., Gryte, C. C. and Studebaker, J. F., 1976, A silicone polymer as a steroid reservoir for enzyme-catalyzed steroid reactions, Biotechnol Bioeng, 18: 1777-1792.

8. Tsai, S., Wu, G. and Chiang, C., 1991, Kinetics of enzymatic hydrolysis of olive oil in biphasic organic-aqueous systems, Biotechnol Bioeng, 38: 761-766.

9. Cremonsei, P., Carrea, G., Ferrara, L. and Antonini, E., 1975, Enzymatic preparation of $20 \beta$-hydroxysteroids in a two-phase system, Biotechnol Bioeng, 17: 1101-1108.

10. Boeren, S. and Laane, C., 1987, Steroid conversion by Flavobacterium dehydrogenans in two-liquid-phase systems, Biotechnol Bioeng, 29: 305-309.

11. Pereira, V., Tigli, H. and Gryte, C. C., 1987, Mathematical analysis of two- phase mass transfer in a batch reactor for the chemical transformation of a steroid, Biotechnol Bioeng, 30: 505-513.

12. Danckwerts, P. V., 1970, Gas-Liquid Reactions, (McGraw-Hill, New York).

13. Kubai, R., Komasawa, I., Otake, T. and Iwasa, M., 1974, Fluid and particle motion in turbulent dispersions-III. Particle-liquid hydrodynamics and mass transfer in turbulent dispersions, Chem Eng Sci, 29: 659-668.

14. Marcus, P. I. and Talalay, P., 1955, On the molecular specificity of steroid-enzyme combinations. The kinetics of $\beta$-hydroxysteroid dehydrogenase, Proc Roy Soc London B, 144: 144-116.

15. Villadsen, J., Michelsen, M. L., 1978, Solution of Differential Equation Models by Polynomial Approximation, (Prentice Hall, New York).

16. Radhakrishnan, K. and Hindmarsh, A. C., 1993, Description and use of LSODE (Reference publication NASA RP-1327. NASA: Washington DC.)

17. Wilke, C. R. and Chang, P., 1955, Correlation of diffusion coefficients in dilute solutions, AIChEJ, 1: 264-270.

18. Michaels, A. S., Wong, P. S. L., Prather, R. and Gale, R. M., 1975, A thermodynamic method for predicting the transport of steroids in polymer matrices, AIChEJ, 21: 1073-1080.

\section{ADDRESS}

Correspondence concerning this paper should be addressed to Dr A. Mehra, Department of Chemical Engineering, Indian Institute of Technology, Bombay, Powai, Bombay 400 076, India. 